\title{
Fuchs's heterochromic cyclitis in congenital ocular toxoplasmosis
}

\author{
E La Heij, A Rothova
}

\begin{abstract}
We report a follow-up after 25 years of a patient with a congenital bilateral ocular toxoplasmosis who developed Fuchs's hetrochromic cyclitis in her left eye. Whether Toxoplasma gondii can cause the development of Fuchs's heterochromic cyclitis, as our case suggests, or whether the iridocyclitis is a secondary ocular response to other agents, is not yet clear.
\end{abstract}

Although Fuchs's heterochromic cyclitis is a distinct disease, the specific cause is still unknown. An association between it and ocular toxoplasmosis is assumed because of the presence of chorioretinal scars in $7 \cdot 5 \%-60 \%$ of patients, which are consistent with a previous intraocular toxoplasmosis. ${ }^{12}$ Apart from these toxoplasma-like scars, non-specific chorioretinal lesions are also described. ${ }^{3}$

Two theories explaining the association between the fundus lesions and Fuchs's cyclitis have been proposed. First, the fundus lesions in these patients are due to a previous toxoplasma infection. Secondly, the scars are of non-toxoplasma origin and may result from antibodies produced during the course of anterior segment inflammation (cross reacting with pigment bearing cells in the uvea and fundus). Until now only sporadic cases of Fuchs's disease with an active toxoplasma lesion or a well documented history of active ocular toxoplasmosis have been reported. ${ }^{2}$ We now report, after a follow-up of 25 years, on a patient with a congenital bilateral ocular toxoplasmosis who developed unilateral Fuchs's heterochromic cyclitis.

\section{Case report}

A 4-week-old female child was seen by an ophthalmologist because of suspected congenital toxoplasmosis. Her mother had had a seroconversion in the last trimester of pregnancy (SabinFeldman dye test titre 1:16384). The patient was prematurely born (gestation time: 31 4/7 weeks, weight $1790 \mathrm{~g})$. Paediatric and neurological examinations revealed no apparent abnormalities. She had a positive Sabin-Feldman dye test (titre 1:256). On ophthalmic examination the child had slight microphthalmus of the right eye (cornea diameter on the right $8 \mathrm{~mm}, 9.5 \mathrm{~mm}$ on the left) and pigment on the lens. No abnormalities were seen in the fundus. At the age of $41 / 2$ months the dye test titre had increased (titre 1:4096) and the patient had developed splenomegaly. An electroencephalogram, made when she was 8 months old, showed a slightly abnormal pattern which could be consistent with a diffuse encephalopathy. We diagnosed con- genital toxoplasmosis on the seroconversion in her mother in the last trimester of pregnancy, the increase in antitoxoplasmal antibodies during the first months of the patients' life, and on the clinical picture.

When the patient was 11 years old she consulted an ophthalmologist because of blurred vision in her left eye. Her visual acuity was 20/60 on the right and $20 / 15$ on the left. In addition to the microphthalmus old atrophic hyperpigmented scars compatible with toxoplasmosis were seen in the right fundus. Examination of her left eye disclosed two active peripheral lesions of focal retinochoroiditis and a mild iritis. Both the Sabin-Feldman dye test (titre 1:64) and the complement binding reaction (titre $1: 4$ ) were low positive. The diagnosis of active ocular toxoplasmosis was made, and the patient was treated with atropine and corticosteroids drops. Owing to the peripheral localisation of the inflammatory focus, systemic therapy was considered to be not required. Within several weeks the retinal lesions sharpened and ultimately became atrophic hyperpigmented scars.

Two years later the patient returned to the ophthalmologist with complaints of floaters in her left eye (visual acuity 20/60 on the right and $20 / 15$ on the left). Ophthalmic examination of the left eye revealed fine white keratic precipitates, scattered on the entire endothelium. Heterochromia with the left iris clearly lighter than the right iris and the absence of synechiae were noted (Fig 1); in addition sporadic cells in the aqueous and evident vitreous opacities were observed. Fundus examination of both eyes showed old known atrophic scars; active lesions were not present (Fig 2). Tests to exclude other causes of uveitis (serum angiotensin converting enzyme, serum lysozyme, serological tests for syphilis, Mantoux test, chest $x$ ray) were all within normal range. Based on the clinical picture our diagnosis was Fuchs's heterochromic cyclitis. From the age of 13 years the patient did

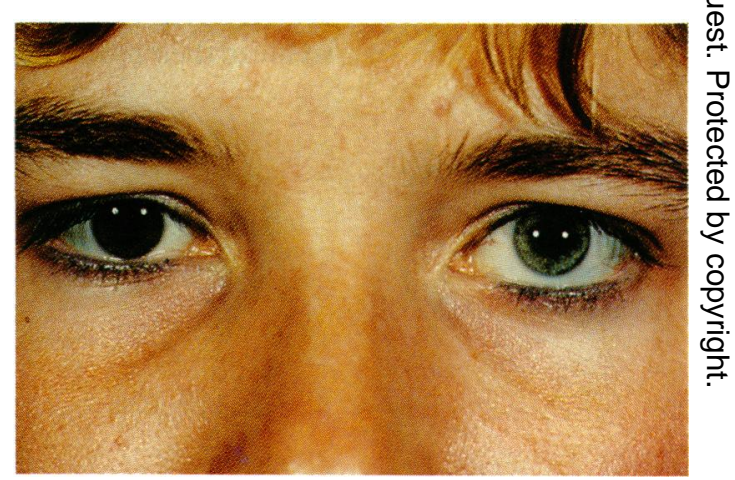

Figure 1 Evident heterochromia in a patient with bilateral congenital ocular toxoplasmosis and Fuchs's heterochromic cyclitis of her left eye. 


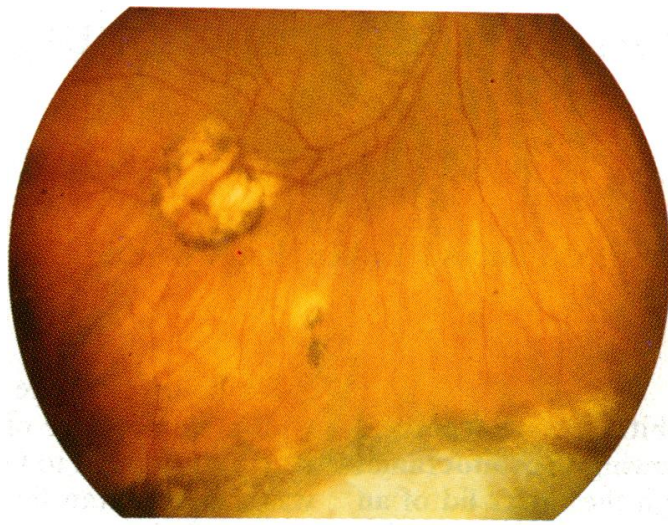

Figure 2A Old atrophic hyperpigmented chorioretinal scars compatible with toxoplasmosis in the right eye.

not suffer another recurrence of ocular toxoplasmosis. Only the fluctuation in intensity of vitreous opacities, characteristic of Fuchs's heterochromic cyclitis, has caused her to visit the ophthalmologist in the past 12 years.

\section{Discussion}

We report this case to help to elucidate the association between Fuchs's heterochromic cyclitis and ocular toxoplasmosis. During a follow-up of 25 years, starting when our patient was 4 weeks old, we recorded the appearance of active toxoplasma lesions in the left retina when the patient was 11 years old and again two years later. A clinical picture characteristic of Fuchs's heterochromic cyclitis then developed in the same eye. Until now only cases of presumptive ocular toxoplasmosis with clinical features of Fuchs's heterochromic cyclitis have been described. This report describes the development of Fuchs's heterochromic cyclitis in a patient with definite congenital ocular toxoplasmosis. The case suggests that infection with Toxoplasma gondii may cause the development of Fuchs's heterochromic cyclitis.

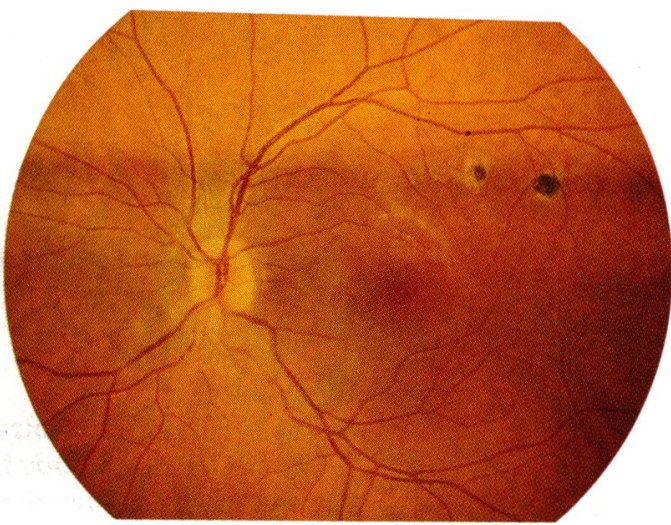

Figure 2B Chorioretinal scars of toxoplasma origin in the left eye with Fuchs's heterochromic cyclitis.

Whether Fuchs's iridocyclitis is a secondary response to a variety of different aetiological agents is not yet clear. ${ }^{4}$ Associations between it and other ocular diseases have been reported retinitis pigmentosa, trauma, scars of non-toxoplasma origin. ${ }^{235}$ Moreover recent immunohistochemical analysis of iris biopsy specimens in these patients has failed to show any specific immunohistological abnormality. ${ }^{6}$ It is therefore conceivable that Fuchs's heterochromic cyclitis may be a single clinical entity with different causes.

1 De Abreu ML, Belfort R, Hirata PS. Fuchs' heterochromic cyclitis and ocular toxoplasmosis. Am $\mathcal{f}$ Ophthalmol 1982; 93: $739-44$.

2 Saraux H, Laroche L, Le Hoang P. Secondary Fuchs' heterochromic cyclitis: a new approach to an old disease. Ophthalmology 1985; 190: 193-8.

3 Arffa RC, Schlaegel TF. Chorioretinal scars in Fuchs' heterochromic iridocyclitis. Arch Ophthalmol 1984; 102: 1153-5.

4 Nussenblatt RB, Palestine AG. Fuchs' heterochromic iridocyclitis. In: Nussenblatt RB, Palestine AG, eds. Uveitis. Fundamentals and clinical practice. Chicago: Year Book Fundamentals and clinical practice. Chicago: Year Book

5 Vourre I, Saari M, Tilikainen A, Rasanen O. Fuchs' heteroourre I, Saari $M$, Tilikainen A, Rasanen $O$. Fuchs' hetero-
chromic cyclitis associated with retinitis pigmentosa: a family study. Can f Ophthalmol 1979; 14: 10-6.

6 Murray PI, Mooy CM, Visser-de Jong E, et al. Immunohistochemical analysis of iris biopsy specimens from patients with Fuchs' heterochromic cyclitis. Am J Ophthalmol 1990; 109: 394-9. 